\title{
Pharmacogenomics of Insulin Secretagogues in Pharmacodynamics, Pharmacokinetics and Adverse Reactions
}

\section{Li Y', Hong $\mathrm{W}^{1,2 *}$ and Li L-C ${ }^{1 *}$}

${ }^{1}$ State Key Laboratory of Cellular Stress Biology, Fujian Provincial Key Laboratory of Innovative Drug Target Research, School of Pharmaceutical Sciences, Xiamen University, Xiamen, China

${ }^{2}$ Institute of Molecular and Cell Biology (IMCB), 61 Biopolis Drive, Singapore

*Corresponding author: Liang-Cheng Li, Associate Professor, State Key Laboratory of Cellular Stress Biology, Fujian Provincial Key Laboratory of Innovative Drug Target Research, School of Pharmaceutical Sciences, Xiamen University, Xiamen, 361102, China

Wanjin Hong, Professor, State Key Laboratory of Cellular Stress Biology, Fujian Provincial Key Laboratory of Innovative Drug Target, School of Pharmaceutical Sciences, Xiamen University, Xiamen, 361102, China; Institute of Molecular and Cell Biology (IMCB), 61 Biopolis Drive, Singapore

Received: August 13, 2021; Accepted: September 15, 2021; Published: September 22, 2021

\section{Abbreviations}

ABCB1: ATP Binding Cassette Subfamily B Member 1; ABCC8: ATP Binding Cassette Subfamily CMember 8; ADR: Adverse Reaction; AUC: Area Under Curve; BCRP: Breast Cancer Resistance Protein; CDKAL1: CDK5 (Cyclin-Dependent Kinase 5) Regulatory Subunit Associated Protein 1-like 1; CDKN2A/2B: Cyclin Dependent Kinase Inhibitor 2A/2B; CYP2C9: Cytochrome P450, Family 2, Subfamily C, Polypeptide 9; CYP2D6: Cytochrome P450, Family 2, Subfamily D, Polypeptide 6; CYP3A4: Cytochrome P450, Family 3, Subfamily A, Polypeptide 4; FPG: Fasting Plasma Glucose; GoDARTS: Genetics of Diabetes Audit and Research Tayside Study; GWAS: Genome Wide Association Study; HbA1c: Glycated Hemoglobin; HOMAIR: Homeostasis Model Assessment-Insulin Resistance; IGF2BP2: Insulin Like Growth Factor 2 MRNA Binding Protein 2; IRS-1: Insulin Receptor Substrate 1; $\mathrm{K}_{\mathrm{ATP}}$ : ATP-sensitive Potassium Channels; KCNJ11: Potassium Inwardly-rectifying Channel, Subfamily J, Member 11; KCNQ1: Potassium Voltage-gated Channel Subfamily Q Member 1; MRP1: Multidrug Resistance-Associated Protein 1; NAMPT: Nicotinamide Phosphoribosyltransferase; NeuroD1: Neuronal Differentiation 1; NIDDM: Non Insulin-dependent Diabetes Mellitus; NOS1AP: Nitric Oxide Synthase 1 Adaptor Protein; OGTT: Oral Glucose Tolerance Test; PAX4: Paired Box 4; PD: Pharmacodynamics; PK: Pharmacokinetics; PPG: Postprandial Plasm Glucose; SLC30A8: Solute Carrier Family 30, Member 8; SLCO1B1: Solute Carrier Organic Anion Transporter Family, Member 1B1; SLCO2B1: Solute Carrier Organic Anion Transporter Family, Member 2B1; SNP: Single Nucleotide Polymorphism; SU:
Sulfonylurea; SUR-1: Sulfonylurea Receptor 1; T2DM: Type 2 Diabetes Mellitus; TCF7L2: T-Cell-Specific Transcription Factor 4; UCP2: Uncoupling Protein 2

\section{Introduction}

Diabetes mellitus has already been a global pandemic and there have been nearly half a billion people suffering from this disease worldwide [1]. With hyperglycemia as the main symptom, diabetes is a serious, chronic condition with dysfunction of glucose, lipid and protein metabolism. The main categories of diabetes are type 1, type 2 and gestational diabetes mellitus. While, Type 2 Diabetes Mellitus (T2DM) is the most common type without clear pathogenesis, which is prevailingly managed by healthy diets and lifestyle, combined with medication if necessary. Insulin secretagogues, including sulfonylureas and glinides, are drugs that can promote the insulin secretion of islet $\beta$-cells to ameliorate hyperglycemia. However, as these medicines are getting more and more extensively used in clinic, some deficiencies emerged: a) interindividual differences of drug efficacy are conspicuous when receiving the same therapy; b) the adverse reactions are severe and unpredictable. Therefore, the studies of pharmacogenomics are the key to understand these problems for better managing the use of these drugs.

Pharmacogenomics is a conception focused on not only the relationship between genetic polymorphism and the efficacy of drugs but also the whole genome and drug development. The polymorphism of coding genes responsible for drug metabolizing enzymes, transporters and drug targets will influence their pharmacokinetics 


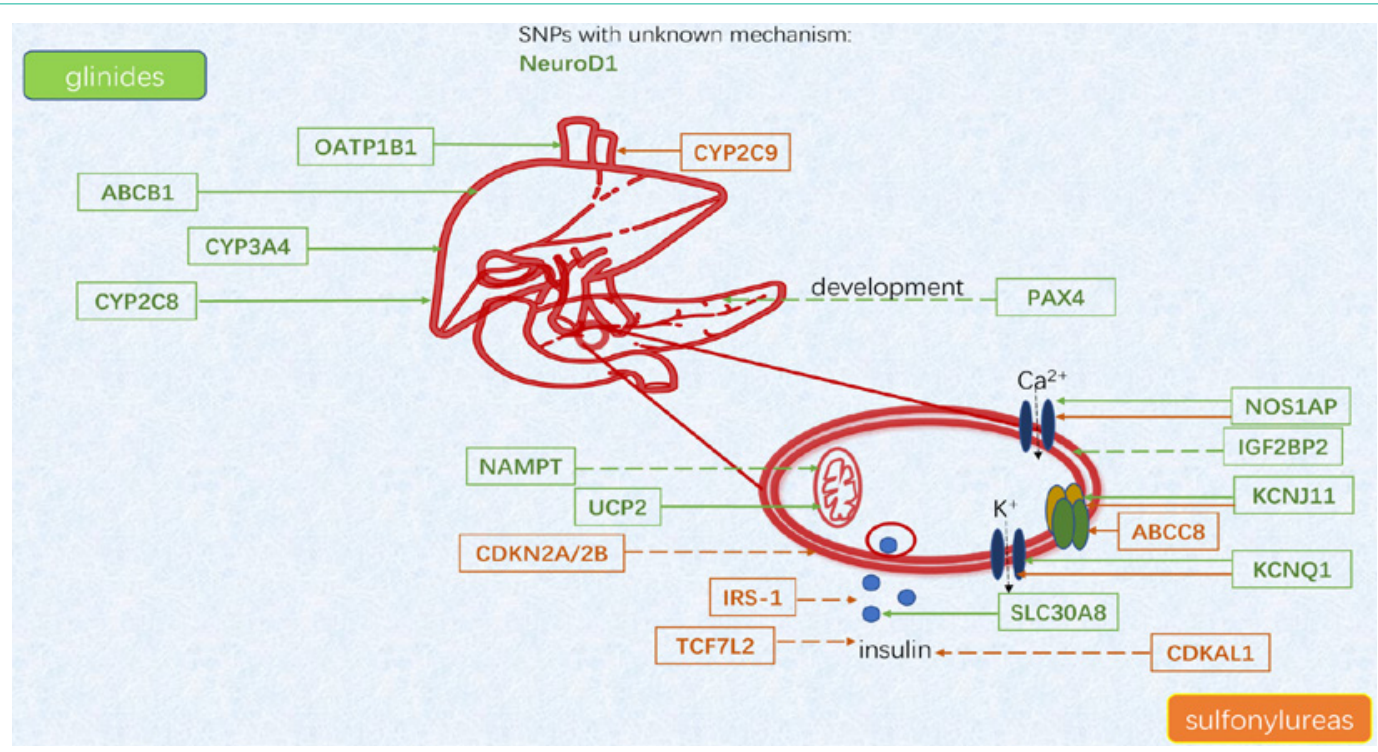

Figure 1: Pharmacogenomics of sulfonylureas and glinides in pharmacokinetics, pharmacodynamics and adverse reactions. Green color refers to glinides and orange color refers to sulfonylureas. The solid lines refer to direct effects and the dotted lines refer to indirect or unknown effects. These polymorphisms cover several certain tissues like liver and pancreas. The specific SNPs of mentioned genes are showed entirely in Supplementary Table 1.

and pharmacodynamics, and eventually leads to the variation of the drug therapeutic efficacy and side effects between individuals [2].

To maximize efficacy of medication and minimize the adverse reactions, it is essential to implement precise medication, which relies on the study of pharmacogenomics. Here, as shown in Figure 1 and Supplementary Table 1, studies of pharmacogenomics on insulin secretagogues, sulfonylureas and glinides, which were published in PubMed between 1999-2020, were searched and cataloged. We are expected to contribute to establishing corresponding assays for personalized medicine and providing information for clinical practice to improve the quality of life and cure rate of T2DM patients.

The efficacy of drugs is a combination of both pharmacokinetics and pharmacodynamics, the changes of pharmacokinetic parameters always lead to the variation of pharmacodynamics, such as the changes of HbA1c, FPG, insulin levels and so on. So, it is difficult to distinguishing whether the effect is caused by pharmacodynamic or pharmacokinetic changes and most of studies not defined clearly. Here, we discuss the pharmacogenomic effects on insulin secretagogues in pharmacodynamics/pharmacokinetics and adverse reactions.

\section{Pharmacogenomics of Sulfonylureas}

Sulfonylureas are recommended by the American Diabetes Association and European Association as second-line agents following metformin monotherapy failure because of the lower cost and good HbAlc-lowing capacity. Sulfonylureas decrease HbAlc levels by facilitating pancreatic $\beta$-cell to secrete insulin. They bind to $K_{\text {ATP }}$ channels of $\beta$-cells, make membrane depolarization, then calcium influx into the cells to trigger insulin release. As a kind of insulin secretagogues, at least $30 \%$ functional $\beta$-cell remaining intact is necessary for sulfonylureas therapy. According to the Genetics of Diabetes Audit and Research in Tayside, Scotland (GoDARTS) study, the failure of sulfonylureas therapy is up to $42.6 \%$ due to genetic variations [3]. Besides, there are many adverse reactions, the most sever one is hypoglycemia.

\section{Pharmacogenomics and pharmacokinetics/ pharmacodynamics of sulfonylureas}

Sulfonylureas are transported by plasma membrane protein SLCO2B1 and then eliminated mainly by hepatic metabolism. MRP1 and BCRP may participate in the transportation of glyburide [4]. However, there is no study showing that the SNPs of MRP1 and BCRP are related with therapeutic effects. Sulfonylureas such as glipizide, glimepiride and tolbutamide are mostly metabolized by the cytochrome p450 2C9(CYP2C9) in the liver. However, CYP3A4 and CYP2C9 contribute to metabolism of glimepiride by $50 \%$ and $30 \%$ respectively. A Genetics of Diabetes Audit and Research Tayside Study (GoDARTS) conducted by Zhou et al. [5] revealed that two CYP2C9 variants-- ${ }^{*}$ (rs1799853) and ${ }^{*} 3$ (rs1057910) are associated with lower enzyme activity and slower metabolism of sulfonylureas. The results suggest that individuals carrying two copies of a loss-offunction allele $\left({ }^{\star} 2 /{ }^{\star} 2\right.$ or ${ }^{\star} 2 /{ }^{\star} 3$ or $\left.{ }^{\star} 3 /{ }^{\star} 3\right)$ were 3.4 times $(\mathrm{P}=0.0009)$ more likely to achieve a treatment $\mathrm{HbA} 1 \mathrm{c}$ level $<7 \%$ than ones with two wild-type CYP2C 9 alleles. Furthermore, ${ }^{\star} 2$ and ${ }^{\star} 3$ allele carriers were less likely to experience treatment failure with sulfonylurea monotherapy $(\mathrm{P}=0.04)$. In conclusion, $C Y P 2 C 9^{\star} 2$ and ${ }^{*} 3$ are kinds of loss-function alleles associated with slower metabolism and higher level of sulfonylurea concentration in the plasma which leads to less failure in view of pharmacokinetics, and better response to sulfonylureas treatment.

It has been clearly known that sulfonylureas target on ATPsensitive potassium channels $\left(\mathrm{K}_{\mathrm{ATP}}\right)$ which consist of four SUR-1 and four inward-rectifier potassium ion channels respectively encoded by $A B C C 8$ and $K C N J 11$. The common variants SNPs of $A B C C 8$ and KCNJ11 are rs757110 and rs5219, rs5210, rs5215 respectively [6]. KCNJ11 and ABCC8 are located on chromosome 11 and only 5kb away from each other, they are linkage disequilibrium inheritance. 
Mutations of KCNJ11 and ABCC8 were proved to cause neonatal diabetes by reducing secretion of insulin. A study by Feng et al. [7] on 1268 Chinese T2DM patients investigated the association between genetic variants and efficacy of gliclazide. Generally, the decrease of Fasting Plasma Glucose (FPG) can be used to test the effect of genetic variants. It was showed that $A B C C 8$ rs757110 and $K C N J 11$ rs5210 were significantly associated with decreasing FPG $(\mathrm{P}=0.002)$. Individuals carrying Ala/Ala genotype significantly had obvious decrease of FPG $(\mathrm{P}<0.001)$ compared to wild-type $(\mathrm{Ser} / \mathrm{Ser})$ after 8 weeks of gliclazide treatment. Besides, individuals carrying Ser/Ala or Ala/Ala genotype significantly had decrease of 2 -h plasma glucose (plasma glucose in 2 hours after OGTT) $(\mathrm{P}=0.001$ and $\mathrm{P}=0.003)$. There were many studies that investigated the relationship between $A B C C 8 / K C N J 11$ and the response to sulfonylureas treatment on different ethnic population, however, some of them were contradictory to each other, which might be impacted by different gene frequencies in different ethnic population or derisory sample size.

Transcription Factor 7 Like 2 (TCF7L2) is one of the earliest genes with many SNPs and is proved to be associated with development of T2DM. It participates in the secretion, proliferation and apoptosis of pancreatic $\beta$-cells as well as the synthesis and process of insulin. Rs7903146 is one of the most studied SNPs of TCF7L2 and is associated with therapeutic effect of sulfonylureas. A GoDARTS study conducted by Pearson et al. [8] illuminated that in rs12255372 variant $\mathrm{T} / \mathrm{T}$ genotype was less likely to respond to sulfonylureas (OR $1.95, \mathrm{P}=0.005$ ) compared with wild-type $\mathrm{G} / \mathrm{G}$. Similarly, in rs7903146 variant $\mathrm{T} / \mathrm{T}$ genotype was less likely to respond to sulfonylureas (OR $1.73, \mathrm{P}=0.015)$ compared with wild-type $\mathrm{C} / \mathrm{C}$, although its effect was not as strong as rs 12255372 . This study consisted of 579 patients treated by sulfonylureas for 12 months. Also, Schroner et al. [9] proved that individuals carrying genotype CC of rs7903146 would have obvious reduction of HbAlc $(\mathrm{P}=0.003)$ and FPG $(\mathrm{P}=0.031)$ in 6 months of sulfonylureas treatment compared with genotype CC.

CDKAL1 (cyclin-dependent kinase 5 (CDK5) regulatory subunit-associated protein 1-like 1) can modulate insulin secretion despite the mechanism is yet to be defined. It has several SNPs, like rs7754840, rs10946398, rs7756992 associated with therapeutic response to sulfonylureas [10]. Schroner et al. [11] conducted a study on 101 T2DM patients who have failed to achieve glycemic control on metformin monotherapy. The end of the trail was FPG levels after 6 months sulfonylureas treatment in different genotypes of CDKAL1 rs7756992. Adjusted the change of FPG $(\triangle \mathrm{FPG})$ was significantly higher in the $\mathrm{AG}+\mathrm{GG}$ compared to the AA group (1.48 \pm 1.51 vs. $1.02 \pm 1.33 \mathrm{mmol} / \mathrm{l}, \mathrm{p}=0.022$ ), while $\mathrm{HbA} 1 \mathrm{c}$ levels were not significantly different. Besides, sulfonylureas doses among three genotype groups were significantly different $(\mathrm{P}=0.032)$ and the $\mathrm{GG}$ genotype received obviously lower dose compared to AA $(\mathrm{P}=0.015)$. In a dominant genetic model, carriers of the $\mathrm{G}$-allele ( $\mathrm{AG}+\mathrm{GG}$ ) achieved significantly lower FPG levels in comparison with patients with AA genotype $(\mathrm{P}=0.013)$. Moreover, a study of relationship between variants of rs7754840 and sulfonylureas resistance was conducted by Soltani et al. [12] in Iranian T2DM patients. There was a significant association between GG genotype and higher risk of sulfonylureas resistance (OR $2.250, \mathrm{P}=0.046$ ). Rs 10946398 was verified to have association with the progress of T2DM but haven't been proved to have association with response to sulfonylureas treatment [10].
KCNQ1 (Potassium Voltage-Gated Channel, KQT-Like Subfamily, Member 1) is wildly expressed in many tissues which plays a role in $\mathrm{K}+$ channel function. Meanwhile, previous GWAS studies in different ethnic population have proved that KCNQ1 has a relationship with T2DM. The mutation of KCNQ1 might lead to impaired insulin secretion. Schroner et al. [13] illustrated $\triangle F P G$ was significantly different among three genotypes (TT, TG and GG) of rs163184 in KCNQ1 $(\mathrm{P}=0.017)$. In a recessive genetic model, carriers of $\mathrm{T}$ allele achieved significant lower FPG levels compared with GG genotype $(\mathrm{P}=0.033) . \triangle \mathrm{FPG}$ was significantly higher in $\mathrm{TT}+\mathrm{TG}$ group than in GG group $(\mathrm{P}=0.016)$. Li et al. [14] expounded the association between rs2237892/rs2237895 and modified-release gliclazide effect in Chinese T2DM patients. In rs2237892 variant, carriers of TT genotype exhibited higher 2-h glucose levels after 16 weeks of sulfonylureas gliclazide MR (modified-release gliclazide) treatment $(\mathrm{P}=0.044)$. whereas, in rs2237895 variant, the $\triangle \mathrm{HbA1c}$ was much higher in $\mathrm{CC}$ group $(\mathrm{P}=0.024)$ compared with $\mathrm{AC}$ group. Compared with the $\mathrm{C}$ allele, the odds ratio for treatment success among carriers of the rs2237892 $\mathrm{T}$ allele was $2.533(\mathrm{P}=0.007)$; and the rs2237895 C allele was associated with a 2.360-fold decrease in HbAlc compared with the A allele $(\mathrm{P}=0.009)$.

The variation of $C D K N 2 A / 2 B$ (Cyclin Dependent Kinase Inhibitor $2 \mathrm{~A} / 2 \mathrm{~B}$ ) was verified to cause dysfunction of pancreatic $\beta$-cells among different population. There was a significant difference in FPG 4 weeks later and $\Delta \mathrm{FPG}$ among $\mathrm{T} / \mathrm{T}, \mathrm{T} / \mathrm{C}$ and $\mathrm{C} / \mathrm{C}$ genotypes in rs10811661 ( $\mathrm{P}=0.025$ and $\mathrm{P}=0.008)$. Carriers of $\mathrm{C}$ allele might have higher response to sulfonylureas according to Ren et al. [15].

NOS1AP (nitric oxide synthase 1 adaptor protein) is involved in the regulation of intracellular $\mathrm{Ca}^{2+}$ levels. Since sulfonylurea promotes insulin secretion by increasing intracellular $\mathrm{Ca}^{2+}$ concentration, Becker et al. [16] conducted a study to illuminate the relationship between NOS1AP and response to sulfonylureas on 619 participants. In participants with the TG or GG genotype at rs10494366 in NOS1AP, glibenclamide was less effective in reducing glucose levels. The mortality rates were also higher compared with glibenclamide users with the TT genotype. In tolbutamide and glimepiride users, patients with the TG and GG genotype were associated with a reduced mortality rate.

The Gly972Ala polymorphism of IRS-1 (insulin receptor substrate-1) was proved to have association with impaired glucosestimulated insulin secretion and resulted in the relative deficiency of insulin [17]. In a study conducted by SESTI et al. [18], the genotype frequency of the Arg972 IRS-1 variant was $8.7 \%$ among diabetic patients well controlled with oral therapy and $16.7 \%$ among patients with secondary failure to sulfonylurea (OR 2.1, $\mathrm{P}=0.01)$.

To summarize, sulfonylureas are metabolized by CYP2C 9 and CYP2C9, and the SNP $* 2$ and $* 3$ are associated with less treatment failure. The target of sulfonylureas is $\mathrm{K}_{\mathrm{ATP}}$, which is encoded by $A B C C 8$ and KCNJ11, and the variants rs757110 and rs5219, rs5210, rs5215 are related to varying decrease of FPG. There are other genes regulating the pharmacodynamic pathways, like TCF7L2 (rs12255372 and rs7903146), CDKAL1 (rs7754840, rs10946398, rs7756992), KCNQ1 (rs2237892 and rs2237895), CDKN2A/2B (rs10811661), NOS1AP (rs10494366) and IRS-1 (Gly972Ala), polymorphisms of which are associated with the therapeutic effect of sulfonylureas by 
influencing the FPG.

\section{Pharmacogenomics and adverse reactions of sulfonylureas}

One of the most severe adverse reaction of sulfonylureas therapy is hypoglycemia, which occurs about $1.8 \%$ patients every year. The cause of hypoglycemia is the slower metabolism of sulfonylureas. Carriers with variants $C Y P 2 C 9^{\star} 2$ and $C Y P 2 C 9^{*} 3$ are slow metabolizers, resulting in higher concentration of sulfonylureas in their plasma than wild type. The higher the drug levels in the plasma are, the more chances of causing adverse reaction. The association between CYP2C9*2/*3 and the risk of sulfonylurea-induced hypoglycemia was elucidated successively by A. Holstein et al. in 2004 [19] and 2010 [20]. Dujic et al. [21] also clarified that variants of CYP2C9 were associated with nearly three-fold higher odds of hypoglycemia (OR $2.81, \mathrm{P}=0.009)$ and better response to $\mathrm{SU}$ treatment $(\mathrm{P}=0.003)$ only in patients carrying the $\mathrm{POR}^{\star} 1 /{ }^{\star} 1$ genotype.

To summarize, the adverse reaction is mainly caused by the excessive concentration of sulfonylureas in the plasma, and it is closely correlated with drug metabolism. The slow metabolizers have better response to sulfonylureas, but on the other hand, they may have more chances of suffering from hypoglycemia.

\section{Pharmacogenomics of Glinides}

Like sulfonylureas, glinides consisting of nateglinide, repaglinide and mitiglinide are also insulin secretagogues which targeting at $\mathrm{K}_{\text {ATP }}$ channels of $\beta$-cells but with different binding sites. It is wildly used to control hyperglycemia after meals because of the rapid acting, that's why it is called prandial glucose regulator. The hypoglycemia can be avoided by monotherapy of glinides, and when combined with metformin, glinides can stabilize glucose levels and reduce the dose of insulin. Nateglinide and repaglinide are effective in reducing the HbA1c levels by $0.4-0.8 \%$ and $1 \%$ respectively in T2DM [22], and mitiglinide is $0.17-1.1 \%$ in reducing HbAlc [23]. Although, glinides are wildly used in clinic because of the safety and efficacy, like other antidiabetic agents, however, the therapeutic effect of glinides also has interindividual difference and the failure of treatment up to $40 \%$.

\section{Pharmacogenomics and pharmacokinetics of repaglinide}

Taking repaglinide as an example, as a kind of hydrophilic agents, repaglinide is first taken up from the blood to hepatocytes by $S L C O 1 B 1$ and then transformed into inactive metabolites via CYP2C8 and CYP3A4 $[24,25]$. But glinides sometimes can be pumped out by $A B C B 1$ which will reduce the concentration of drug in target cells. Glinides are metabolized mainly by hepatic cytochrome P450 (CYP450). E.g. repaglinide is metabolized by CYP2C8 and CYP3A4 [26], while nateglinide is primarily metabolized by $C Y P 2 C 9$ and to a lesser extent by CYP3A4 and CYP2D6 respectively [27].

Variants of CYP2C8 and CYP3A4 have an impact on clearance rates of repaglinide, carriers with $C Y P 2 C 8^{\star} 1 /^{\star} 1$ had greater AUC than others with $C Y P 2 C 8^{\star} 1 /{ }^{*} 3$ in Caucasians population $[28-30]$ and CYP $3 A 4^{*} 1{ }^{*} 18$ was associated with lower elimination rate $(44.0 \%)$ than $C Y P 3 A 4^{\star} 1 /^{\star} 1$ in Malaysian population [31]. However, for nateglinide, $C Y P 2 C 9^{*} 3$ displayed significantly reduction in clearance and higher AUC $[32,33]$ while $C Y P 2 D 6^{\star} 4$ and $C Y P 2 D 6^{\star} 5$ showed no significant effect on nateglinide clearance [32].
SLCO1B1 or OATP1B (Solute Carrier Organic Anion Transporter Family Member 1B1) is influx transporter that absorbs repaglinide and nateglinide from the plasma into hepatocytes. Carriers with CC or CT genotype of rs4149056 in SLCO1B1 had greater AUC than others with TT genotype due to the less transport activity [28,34-37].

ABCB1 (ATP Binding Cassette Subfamily B Member 1) or MDR1 is efflux transporter that pump repaglinide out of cells to decrease the concentration in target cells. For repaglinide, T allele of rs2032582 in $M D R 1$ was associated with higher levels of repaglinide which signified higher response $(\mathrm{P}=0.007)$ [38].

To summarize, the variants of CYP2C $8\left({ }^{\star} 3\right) / C Y P 3 A 4\left({ }^{*} 18\right)$, SLCO1B1 (rs4149056) and ABCB1 (rs2032582) impact the pharmacokinetics of glinides to vary the concentration in plasma and targets sits.

\section{Pharmacogenomics and pharmacodynamics of repaglinide}

Both sulfonylureas and repaglinide act on $\mathrm{K}_{\mathrm{ATP}}$ channels, so the effects of variants may be similar to some extent although they target at different subunits of the channels.

Rs2237892/rs2237895 of KCNQ1 [39], rs10494366 of NOS1AP [40] and rs5219 of KCNJ11 [41] were found associated with effect of both repaglinide and sulfonylureas. The relationship between sulfonylureas and aforementioned variants was already shown previously and the effects were same. It is remarkable that variant rs10494366 of NOS1AP was significantly correlated with relieving insulin resistance. It was reported that the variant of rs10494366 and repaglinide treatment had an interaction effect only in HOMA-IR $(\mathrm{P}=0.013)$, indicating that TT genotype may associated with insulin resistance. There was a significant difference in the response rate to repaglinide treatment between the $\mathrm{E}$ and $\mathrm{K}$ alleles of rs5219 in KCNJ11 (68\% vs. $82 \%, \mathrm{P}=0.0324$ ).

Rs290287 is a SNP of TCF7L2 that is associated with response to repaglinide according to $\mathrm{Yu}$ et al. [42]. The study exhibited that TT homozygotes showed greater efficacy on the levels of fasting insulin, triglycerides and low-density lipoprotein cholesterol than $\mathrm{C}$ allele carriers.

SLC30A8 or $Z n T-8$, encoding a zinc transporter, is expressed at a high level only in the pancreas whose variants are certainly associated with developing type 2 diabetes mellitus. It pumps zinc into vesicles of insulin from endochylema and takes part in insulin secretion. Two of its variants-rs 13266634 and rs16889462 were also correlated with repaglinide treatment. T allele of rs13266634 and GA genotype of rs16889462 both showed enhanced response to repaglinide according to $\mathrm{Wu}$ et al. [43].

IGF2BP2 (Insulin like Growth Factor 2 MRNA Binding Protein 2) regulates the translation of IGF-2, which plays an important role in growth and insulin signaling. Rs1470579 and rs4402960 are identified to be associated with the effect of repaglinide treatment. C allele carriers had lower effects of repaglinide treatment on reducing FPG $(\mathrm{P}<0.05)$ and PPG $(\mathrm{P}<0.05)$ than AA homozygotes, while $\mathrm{T}$ allele of rs4402960 carriers displayed an enhanced effect than GG homozygotes $(\mathrm{P}<0.01)[44]$.

UCP2 (Uncoupling Protein 2), also refer to $S L C 25 A 8$, is related 
to glucose-stimulated insulin secretion. Rs659366 is associated with the effect of repaglinide according to Wang et al. [33,45] A allele was associated with smaller decrease in FPG $(\mathrm{P}<0.05)$ and $\mathrm{HbAlc}$ $(\mathrm{P}<0.05)$ levels compared to GG genotype.

NAMPT (Nicotinamide Phosphoribosyltransferase) encodes systemic NAD biosynthetic enzyme that regulates insulin secretion. Variant rs11977021 has influences on repaglinide efficacy through FPG, PPG and HbAlc levels as evidenced by a study by Shang et al. [46].

To summarize, like sulfonylureas, glinides also target on $\mathrm{K}_{\mathrm{ATP}}$ but on different subunits. Therefore, the same variants (rs2237892/ rs2237895 of KCNQ1, rs10494366 of NOS1AP and rs5219 of KCNJ11) also have influence on the therapeutic effect of glinides. The pharmacodynamics of glinides is relevant to the synthesis and secretion of insulin, the development of pancreatic islets and the insulin signaling. The correlated genes like TCF7L2 (rs290287), SLC30A8 (rs13266634 and rs16889462), IGF2BP2 (rs1470579 and rs4402960), UCP2 (rs659366) and NAMPT (rs11977021) contribute to varying $\mathrm{FPG} / \mathrm{PPG}$ or $\mathrm{HbA1c}$ levels.

\section{Pharmacogenomics and adverse reactions of glinides}

Because of the shorter half-life of glinides, there is lower risk of treatment-related hypoglycemia. But compared with sulfonylureas, glinides have more chances of having weight gain [47]. Although there is no study directly probing the association between adverse reactions and glinides treatment, we still can make a hypothesis that hypoglycemia may be associated with higher concentration of drug in the plasma due to slower elimination of drugs. Therefore, the individuals with decreased function of CYP450 (slow metabolizer) are likely have more chances of developing hypoglycemia during the treatment. As for weight gain, there still remains controversy.

\section{Conclusion and Future Perspective}

Insulin secretagogues are widely used in clinic to control hyperglycemia, but their adverse reactions and individual variation still can't be neglected. Sulfonylureas and glinides are recommended as second-line medicines, likely because of more adverse reactions such as hypoglycemia. The diagnosis of genotypes of patients is also essential for personalized medicine. Sulfonylureas are mostly metabolized by CYP2C9 and CYP3A4, patients with decreased function of these genes will have better effects with lower dosage but the chances of hypoglycemia are also increased at the same time. Moreover, variants of ABCC8, KCNJ11, TCF7L2, CDKAL1, KCNQ1, $C D K N 2 A / 2 B, N O S 1 A P$ and IRS- 1 are significantly associated with the response to sulfonylureas by impacting the pharmacodynamics.

As for glinides, although they are safer than sulfonylureas, but their treatment failure can't be neglected. Except variants of KCNQ1, NOS1AP, TCF7L2 and KCNJ11 are both associated with response to sulfonylureas and glinides, IGF2BP2, UCP2 and NAMPT are correlated with pharmacodynamics of repaglinide as they play important roles in drugs' efficacy. Furthermore, the main metabolic enzymes such as CYP2C 8 and CYP3A4 also contribute to therapeutic efficacy and adverse reaction by regulated drug concentration in the plasma.

In conclusion, there is still a long way to explicitly define the relationship between genetic variation and therapeutic effect of medications. Only by conducting more GWAS studies, to deep understanding the effect of genetic variation on drug action, we can design individualized therapy, which will improve therapy efficacy and decrease adverse reactions.

\section{Declaration}

Acknowledgments: This work was supported by National Natural Science Foundation of China (82073908), and in part by The Natural Science Foundation of Fujian Province (2020J01041) to Liang-cheng $\mathrm{Li}$, and by funding from the Fundamental Research Funds for the Central Universities (20720212004) and XMU Training Program of Innovation and Entrepreneurship for Undergraduates (202110384281) to Yu-jie Li.

Contribution statement: Y.J.L drafted the manuscript. W.J.H and L.L.C. conceived the project, and wrote the manuscript.

\section{References}

1. IDF DIABETES ATLAS Ninth edition 2019. 2019.

2. Heo $\mathrm{CU}$ and $\mathrm{Cl}$ Choi. Current Progress in Pharmacogenetics of Second-Line Antidiabetic Medications: Towards Precision Medicine for Type 2 Diabetes. J Clin Med. 2019; 8.

3. Loganadan NK, HZ Huri, SR Vethakkan and Z Hussein. Clinical and genetic predictors of secondary sulfonylurea failure in Type 2 diabetes patients: the SUCLINGEN study. Pharmacogenomics. 2020; 21: 587-600.

4. Holstein A and W Beil. Oral antidiabetic drug metabolism: pharmacogenomics and drug interactions. Expert Opin Drug Metab Toxicol. 2009; 5: 225-241.

5. Zhou K, L Donnelly, L Burch, R Tavendale, AS Doney, G Leese, et al. Lossof-function CYP2C9 variants improve therapeutic response to sulfonylureas in type 2 diabetes: a Go-DARTS study. Clin Pharmacol Ther. 2010; 87: 52-56.

6. Loganadan NK, HZ Huri, SR Vethakkan and Z Hussein. Genetic markers predicting sulphonylurea treatment outcomes in type 2 diabetes patients: current evidence and challenges for clinical implementation. Pharmacogenomics J. 2016; 16: 209-219.

7. Feng $\mathrm{Y}, \mathrm{G}$ Mao, $\mathrm{X}$ Ren, $\mathrm{H}$ Xing, G Tang, Q Li, et al. Ser1369Ala variant in sulfonylurea receptor gene $A B C C 8$ is associated with antidiabetic efficacy of gliclazide in Chinese type 2 diabetic patients. Diabetes Care. 2008; 31: 1939-1944

8. Pearson ER, LA Donnelly, C Kimber, A Whitley, AS Doney, MI McCarthy, et al. Variation in TCF7L2 influences therapeutic response to sulfonylureas: a GoDARTs study. Diabetes. 2007; 56: 2178-2182.

9. Schroner, Z, M Javorsky, R Tkacova, L Klimcakova, M Dobrikova, V Habalova, et al. Effect of sulphonylurea treatment on glycaemic control is related to TCF7L2 genotype in patients with type 2 diabetes. Diabetes, Obesity and Metabolism. 2011; 13: 89-91.

10. Chistiakov DA, VA Potapov, SA Smetanina, LN Bel'chikova, LA Suplotova and VV Nosikov. The carriage of risk variants of CDKAL1 impairs beta-cell function in both diabetic and non-diabetic patients and reduces response to non-sulfonylurea and sulfonylurea agonists of the pancreatic $\mathrm{K}_{\text {ATP }}$ channel. Acta Diabetol, 2011; 48: 227-235.

11. Schroner Z, M Javorsky J Haluskova, L Klimcakova, E Babjakova, M Fabianova, et al. Variation in CDKAL1 gene is associated with therapeutic response to sulphonylureas. Physiol Res. 2012; 61: 177-183.

12. Soltani G, Z Hatefi, AR Salehi, S Khosravi, MR Ghiasi, K Teke, et al. Pharmacogenomics of Sulfonylureas Response in Relation to rs7754840 Polymorphisms in Cyclin-Dependent Kinase 5 Regulatory Subunit-associated Protein 1-like (CDKAL1) Gene in Iranian Type 2 Diabetes Patients. Adv Biomed Res. 2018; 7: 96.

13. Zbynek S, D Martina, K Lucia, J Martin, Z Jozef, K Miriam, et al. Variation in $K C N Q 1$ is associated with therapeutic response to sulphonylureas. Med Sci Monit. 2011; 17: 392-396. 
14. Li Q, TT Tang, F Jiang, R Zhang, M Chen, J Yin, et al. Polymorphisms of the $K C N Q 1$ gene are associated with the therapeutic responses of sulfonylureas in Chinese patients with type 2 diabetes. Acta Pharmacol Sin. 2017; 38: 8089.

15. Ren Q, X Han, Y Tang, X Zhang, X Zou, X Cai, et al. Search for genetic determinants of sulfonylurea efficacy in type 2 diabetic patients from China Diabetologia. 2014; 57: 746-753.

16. Beckera, ML, A-JLHJ. Aarnoudsea, C Newton-Chehe, A Hofmana, JCM Wittemana, AG Uitterlindena, et al. Common variation in the NOS1AP gene is associated with reduced glucose-lowering effect and with increased mortality in users of sulfonylurea. Pharmacogenetics and Genomics. 2008; 18: 591 594.

17. Ottavia P, F Massimo, L Marta, Hribal, L Davide, A Domenico, L Renato, et al. The Gly972 $\rightarrow$ Arg amino acid polymorphism in IRS-1 impairs insulin secretion in pancreatic $\beta$ cells. J. Clin. Invest. 1999; 104: 357-364.

18. Giorgio S, MA Marini, M Cardellini, A Sciacqua, S Frontoni, PF Andreozzi, et al. The Arg972 Variant in Insulin Receptor Substrate-1 Is Associated With an Increased Risk of Secondary Failure to Sulfonylurea in Patients With Type 2 Diabetes. Diabetes Care. 2004; 27: 1394-1398.

19. Holstein A, A Plaschke M Ptak, EH Egberts, J El-Din, J Brockmoller, et al. Association between CYP2C9 slow metabolizer genotypes and severe hypoglycaemia on medication with sulphonylurea hypoglycaemic agents. $\mathrm{Br}$ J Clin Pharmacol. 2005; 60: 103-106.

20. Holstein A, M Hahn, O Patzer, A Seeringer, P Kovacs and J Stingl. Impact of clinical factors and CYP2C9 variants for the risk of severe sulfonylureainduced hypoglycemia. Eur J Clin Pharmacol. 2011; 67: 471-476.

21. Dujic T, K Zhou, LA Donnelly, G Leese, CNA Palmer and ER Pearson Interaction between variants in the CYP2C9 and POR genes and the risk of sulfonylurea-induced hypoglycaemia: A GoDARTS Study. Diabetes Obes Metab. 2018; 20: 211-214.

22. Guardado-Mendoza R, A Prioletta LM Jiménez-Ceja, A Sosale and F Folli. The role of nateglinide and repaglinide, derivatives of meglitinide, in the treatment of type 2 diabetes mellitus. Arch Med Sci. 2013; 9: 936-943.

23. Phillippe HM and KA Wargo. Mitiglinide for type 2 diabetes treatment. Expert Opin Pharmacother. 2013; 14: 2133-2144.

24. König J, Y Cui, AT Nies and D Keppler. A novel human organic anion transporting polypeptide localized to the basolateral hepatocyte membrane. Am J Physiol Gastrointest Liver Physiol. 2000; 278: G156-G164.

25. Bidstrup TB, I Bjørnsdottir, UG Sidelmann, MS Thomsen and KT Hansen, CYP2C8 and CYP3A4 are the principal enzymes involved in the human in vitro biotransformation of the insulin secretagogue repaglinide. $\mathrm{Br} \mathrm{J} \mathrm{Clin}$ Pharmacol. 2003; 56: 305-314.

26. Vibeke H. Clinical Pharmacokinetics and Pharmacodynamics of Repaglinide. Clin Pharmacokinet. 2002; 41: 471-483.

27. Miao C, H Cheng and J Weiping. Pharmacogenomics of glinides. Pharmacogenomics. 2015; 16: 45-60.

28. Niemi M, JT Backman, LI Kajosaari, JB Leathart, M Neuvonen, AK Daly, et al. Polymorphic organic anion transporting polypeptide $1 \mathrm{~B} 1$ is a majo determinant of repaglinide pharmacokinetics. Clin Pharmacol Ther. 2005; 77 468-478.

29. Niemi M, JB Leathart, M Neuvonen, JT Backman, AK Daly, and PJ Neuvonen. Polymorphism in CYP2C8 is associated with reduced plasma concentrations of repaglinide. Clinical Pharmacology \& Therapeutics. 2003; 74: 380-387.

30. Rodriguez-Antona C, M Niemi JT Backman, LI Kajosaari, PJ Neuvonen, $\mathrm{M}$ Robledo, et al. Characterization of novel CYP2C8 haplotypes and thei contribution to paclitaxel and repaglinide metabolism. Pharmacogenomics J. 2008; 8: 268-277.
31. Ruzilawati $A B$ and SH Gan. CYP3A4 genetic polymorphism influences repaglinide's pharmacokinetics. Pharmacology. 2010; 85: 357-364

32. Julia K, M Ingolf, Ml Go ran, B Steffen, R Wolfgang, M Christian, et al Influence of CYP2C9 and CYP2D6 Polymorphisms on the Pharmacokinetics of Nateglinide in Genotyped Healthy Volunteers. Clin Pharmacokinet. 2004; 43: $267-278$

33. Cheng $Y, G$ Wang, $W$ Zhang, L Fan, $Y$ Chen, and HH Zhou. Effect of CYP2C9 and SLCO1B1 polymorphisms on the pharmacokinetics and pharmacodynamics of nateglinide in healthy Chinese male volunteers. Eur J Clin Pharmacol. 2013; 69: 407-413.

34. Kalliokoski A, M Neuvonen, PJ Neuvonen and M Niemi. Different effects of SLCO1B1 polymorphism on the pharmacokinetics and pharmacodynamics of repaglinide and nateglinide. J Clin Pharmacol. 2008; 48: 311-321.

35. Zhang W, YJ He, CT Han, ZQ Liu, Q Li, L Fan, et al. Effect of SLCO1B1 genetic polymorphism on the pharmacokinetics of nateglinide. $\mathrm{Br} \mathrm{J} \mathrm{Clin}$ Pharmacol. 2006; 62: 567-572.

36. Kalliokoski A, M Neuvonen, PJ Neuvonen and M Niemi. The effect of SLCO1B1 polymorphism on repaglinide pharmacokinetics persists over a wide dose range. Br J Clin Pharmacol. 2008; 66: 818-825.

37. He J, Z Qiu, N Li, Y Yu, Y Lu, D Han, et al. Effects of SLCO1B1 polymorphisms on the pharmacokinetics and pharmacodynamics of repaglinide in healthy Chinese volunteers. Eur J Clin Pharmacol. 2011; 67: 701-707.

38. Xiang $Q$, YM Cui, $X$ Zhao, $L$ Yan and $Y$ Zhou. The Influence of MDR1 G2677T/a genetic polymorphisms on the pharmacokinetics of repaglinide in healthy Chinese volunteers. Pharmacology. 2012; 89: 105-110.

39. Yu W, C Hu, R Zhang, C Wang, W Qin, J Lu, et al. Effects of KCNQ1 polymorphisms on the therapeutic efficacy of oral antidiabetic drugs in Chinese patients with type 2 diabetes. Clin Pharmacol Ther. 2011; 89: 437 442

40. Qin W, R Zhang, C Hu, CR Wang, JY Lu, WH Yu, et al. A variation in NOS1AP gene is associated with repaglinide efficacy on insulin resistance in type 2 diabetes of Chinese. Acta Pharmacol Sin. 2010; 31: 450-454.

41. He YY, R Zhang, XY Shao, C Hu, CR Wang, JX Lu, et al. Association of $K C N J 11$ and $A B C C 8$ genetic polymorphisms with response to repaglinide in Chinese diabetic patients. Acta Pharmacol Sin. 2008; 29: 983-989.

42. Yu M, XJ Xu, JY Yin, J Wu, X Chen, ZC Gong, et al. KCNJ11 Lys23Glu and TCF7L2 rs290487(C/T) polymorphisms affect therapeutic efficacy of repaglinide in Chinese patients with type 2 diabetes. Clin Pharmacol Ther. 2010; 87: 330-335

43. Wu Y, H Li, RJ Loos, Z Yu, X Ye, L Chen, et al. Common variants in CDKAL1, CDKN2A/B, IGF2BP2, SLC3OA8, and HHEX/IDE genes are associated with type 2 diabetes and impaired fasting glucose in a Chinese Han population. Diabetes. 2008; 57: 2834-2842.

44. Huang Q, JY Yin, XP Dai, Q Pei, M Dong, ZG Zhou, et al. IGF2BP2 variations influence repaglinide response and risk of type 2 diabetes in Chinese population. Acta Pharmacol Sin. 2010; 31: 709-717.

45. Wang S, YM Se, ZQ Liu, MX Lei, B Hao, ZX Sun, et al. Effect of genetic polymorphism of UCP2-866 G/A on repaglinide response in Chinese patients with type 2 diabetes. Pharmazie. 2012; 67: 74-79.

46. Sheng FF, XP Dai, J Qu, GH Lei, HB Lu, J Wu, et al. NAMPT -3186C/T polymorphism affects repaglinide response in Chinese patients with Type 2 diabetes mellitus. Clin Exp Pharmacol Physiol. 2011; 38: 550-554.

47. Plosker GL and DP Figgitt. Repaglinide A Pharmacoeconomic Review of its Use in Type 2 Diabetes Mellitus. Pharmacoeconomics. 2004: 389-411. 